\title{
On the Study of Second-Order Wave Theory and Its Convergence for a Two-Fluid System
}

\author{
Chi-Min Liu,, ${ }^{1,2}$ Hwung-Hweng Hwung, ${ }^{2,3}$ and Ray-Yeng Yang ${ }^{2,4}$ \\ ${ }^{1}$ Division of Mathematics, General Education Center, Chienkuo Technology University, Changhua city 500, Taiwan \\ ${ }^{2}$ International Wave Dynamics Research Center, National Cheng Kung University, Tainan 701, Taiwan \\ ${ }^{3}$ Department of Hydraulic and Ocean Engineering, National Cheng Kung University, Tainan 701, Taiwan \\ ${ }^{4}$ Tainan Hydraulic Laboratory, National Cheng Kung University, Tainan 701, Taiwan
}

Correspondence should be addressed to Chi-Min Liu; cmliu@ctu.edu.tw

Received 24 December 2012; Accepted 19 March 2013

Academic Editor: Kuppalapalle Vajravelu

Copyright (C) 2013 Chi-Min Liu et al. This is an open access article distributed under the Creative Commons Attribution License, which permits unrestricted use, distribution, and reproduction in any medium, provided the original work is properly cited.

\begin{abstract}
Second-order solutions of internal and surface waves in a two-fluid system are theoretically analyzed in this study. Using the perturbation technique, the derivation of second-order solutions for internal waves is revisited, and the results are expressed in one-by-one forms instead of a matrix form. Second-order solutions arising from the interactions of two arbitrary linear waves of different frequencies contain the sum-frequency (superharmonic) and the difference-frequency (subharmonic) components, which are separately examined. Internal Stokes wave being a special case of present solutions is firstly investigated. Next, the convergence of second-order theory and the second-order effects on wave profiles are analyzed. For general cases, the effects of the thickness ratio of two fluids and the ratio of wavenumbers of two first-order waves on second-order wave characteristics, which include transfer functions and particle velocities, are also examined. Moreover, most existing theories for the one-fluid and two-fluid systems can be deduced from present solutions.
\end{abstract}

\section{Introduction}

A natural wave train is usually composed of thousands of waves with different frequencies. Second-order waves are generated due to the interaction between two arbitrary linear waves. Hence, there are two types of second-order components, the sum-frequency and the difference-frequency components. These two types of second-order terms are the so-called superharmonic and subharmonic components. For second-order wave theories, most of the past studies focused on waves propagating over a single-layer fluid. For sinusoidal waves at first order, Stokes [1] provided secondorder solutions describing the self-interaction of first-order waves using wave steepness as a perturbation parameter. An important conclusion is that the wave profile has a sharper crest and a flatter trough rather than a simple sine wave due to second-order effects. Stokes' theory not only plays an important role in high-order analyses of sinusoidal waves but also virtually forms a foundation for further nonlinear wave studies. It is, however, merely a special case of superharmonic interactions. For random waves with different frequencies and wavelengths, super- and subharmonic waves are naturally generated to form more complicated wave profiles rather than Stokes waves. With the restriction of slightly different frequencies (the narrow-band restriction), Longuet-Higgins and Stewart [2, 3] provided solutions for the subharmonic interactions. Ottesen-Hansen [4] overcame the narrow-band restriction of frequencies and represented the expressions of subharmonic waves in a more adequate form with the introduction of transfer functions, which are defined as the ratio of the second-order amplitude to the first-order amplitudes of waves. Hence, the application of transfer functions enables one to easily measure the secondorder effects on wave profiles. Dean and Sharma [5] gave a unified and more compact theory for both superharmonic and subharmonic waves. In practical applications, the theory of second-order surface waves is frequently applied to design the signals of wavemakers $[6,7]$.

Though the results for second-order surface waves are abundant, studies on second-order internal waves are 
comparatively few. Hunt [8] provided the solutions for internal Stokes waves in a two-fluid system with a rigid lid. Thorpe [9] further examined internal Stokes waves and discussed a number of cases with various thickness ratios and relative wave lengths. He also carried out a comparison between experimental measurements and theoretical results. Tsuji and Nagata [10] applied Stokes theory to study the fifth-order superharmonic interfacial waves between two-layer fluids of infinite thicknesses. This was later checked by Holyer [11] who numerically calculated the series of internal waves to the 31st order using the Fourier expansion procedure. Umeyama [12] theoretically and experimentally studied third-order Stokes waves for slow-mode motion. His derived solution and experimental results are quite coincident. Though internal Stokes wave theory has been widely developed in above studies, it seems that other superharmonic and subharmonic interactions are rarely stressed. Later, Song [13] derived the superharmonic and subharmonic solutions for random internal waves in a two-fluid system bounded by two rigid plates. In oceans, a very slight difference in densities across the pycnocline leads to an infinitesimal displacement at the free surface; the rigid upper boundary is reasonably assumed to be rigid in order to simplify the theoretical analysis without a great loss of accuracy. Liu [14] removed this rigid restriction on the free surface and theoretically derived the secondorder super- and subharmonic solutions. The solutions are expressed in matrix form. In practice, his results can be applied to a considerably wide range of density ratios making his solutions superior to Song's solutions. Preliminary investigations of transfer functions were also addressed in his study.

The main purpose of the present paper is to theoretically examine the convergence of second-order wave theory and the second-order effects on wave properties. In Section 2, using the perturbation technique, the derivation of secondorder solutions for internal waves is revisited and the results are expressed in a one-by-one form instead of a matrix form [14]. Superharmonic and subharmonic interactions are, respectively, examined. In addition, most existing theories for the two-fluid and one-fluid systems are included in the present solutions. Wave characteristics of the slowmode motion are analyzed. In Section 3, the internal Stokes wave being a special case of superharmonic interactions is investigated. The convergence range for the second-order theory is studied. All second-order wave properties should be subject to this convergence range to ensure the perturbation valid. Wave profiles influenced by second-order effects are subsequently examined. Interactions arising from two arbitrary linear waves are investigated in Section 4. Wave characteristics including the convergence range, transfer functions, and particle velocities are studied. Conclusions are presented in Section 5.

\section{Review of the Second-Order Theory for a Two-Fluid System}

In this section, the second-order solutions of internal waves in a two-fluid system are briefly revisited. The schematic diagram is shown in Figure 1. General assumptions and

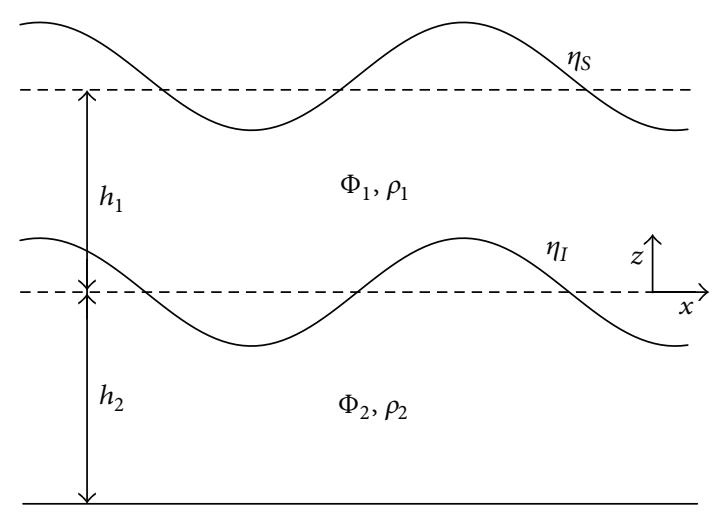

Figure 1: Definition sketch.

definitions of symbols are described as follows. The flow in each layer is assumed to be irrotational and inviscid. $\Phi_{1}$ and $\Phi_{2}$ are the velocity potentials of the upper- and lowerlayer fluids, respectively. $\rho_{r}$ describes the density ratio of the upper fluid $\rho_{1}$ relative to the lower fluid $\rho_{2} . h_{1}$ and $h_{2}$ stand for the undisturbed thicknesses of the upper and the lower layers, respectively. The displacements of the free surface and the interface are represented as $\eta_{S}$ and $\eta_{I}$, respectively. The horizontal coordinate is denoted by $x$, and $z$ represents the vertical coordinate originating at the undisturbed interface and pointing upward. Hence, the governing equations and boundary conditions are expressed as

$$
\begin{aligned}
& \frac{\partial^{2} \Phi_{1}}{\partial x^{2}}+\frac{\partial^{2} \Phi_{1}}{\partial z^{2}}=0 \\
& \frac{\partial^{2} \Phi_{2}}{\partial x^{2}}+\frac{\partial^{2} \Phi_{2}}{\partial z^{2}}=0 \\
& \frac{\partial \Phi_{1}}{\partial z}=\frac{\partial \eta_{I}}{\partial t}+\frac{\partial \Phi_{1}}{\partial x} \frac{\partial \eta_{I}}{\partial x} \quad \text { at } z=\eta_{I} \\
& \frac{\partial \Phi_{2}}{\partial z}=\frac{\partial \eta_{I}}{\partial t}+\frac{\partial \Phi_{2}}{\partial x} \frac{\partial \eta_{I}}{\partial x} \quad \text { at } z=\eta_{I} \\
& \rho_{r}\left[g \eta_{I}+\frac{\partial \Phi_{1}}{\partial t}+\frac{1}{2}\left(\frac{\partial \Phi_{1}}{\partial x}\right)^{2}+\frac{1}{2}\left(\frac{\partial \Phi_{1}}{\partial z}\right)^{2}\right] \\
& =\left[g \eta_{I}+\frac{\partial \Phi_{2}}{\partial t}+\frac{1}{2}\left(\frac{\partial \Phi_{2}}{\partial x}\right)^{2}+\frac{1}{2}\left(\frac{\partial \Phi_{2}}{\partial z}\right)^{2}\right] \quad \text { at } z=\eta_{I} \text {, } \\
& \frac{\partial \Phi_{1}}{\partial z}=\frac{\partial \eta_{S}}{\partial t}+\frac{\partial \Phi_{1}}{\partial x} \frac{\partial \eta_{S}}{\partial x} \quad \text { at } z=h_{1}+\eta_{S} \\
& g \eta_{S}+\frac{\partial \Phi_{1}}{\partial t}+\frac{1}{2}\left(\frac{\partial \Phi_{1}}{\partial x}\right)^{2}+\frac{1}{2}\left(\frac{\partial \Phi_{1}}{\partial z}\right)^{2}=0 \text { at } z=h_{1}+\eta_{S} \text {, } \\
& \frac{\partial \Phi_{2}}{\partial z}=0 \quad \text { at } z=-h_{2} .
\end{aligned}
$$


Next, velocity potentials and displacements of the free surface and the interface are expanded in powers of the wave slope $\varepsilon$ as shown below

$$
\begin{gathered}
\Phi_{1}=\Phi_{1}^{(1)}+\Phi_{1}^{(2)}+\cdots, \\
\Phi_{2}=\Phi_{2}^{(1)}+\Phi_{2}^{(2)}+\cdots, \\
\eta_{I}=\eta_{I}^{(1)}+\eta_{I}^{(2)}+\cdots, \\
\eta_{S}=\eta_{S}^{(1)}+\eta_{S}^{(2)}+\cdots,
\end{gathered}
$$

where the superscripts indicate the order of magnitude in powers of $\varepsilon$, that is, $\Phi_{1}^{(1)}=O(\varepsilon), \Phi_{1}^{(2)}=O\left(\varepsilon^{2}\right)$, and so forth. Using the Taylor-series expansion, the boundary conditions on $z=\eta_{I}$ and $z=h_{1}+\eta_{S}((3)$ to (7)) are, respectively, expanded about $z=0$ and $z=h_{1}$. Accordingly, the amplitudes of internal and surface waves must be small enough to make this expansion valid. Hence, governing equations and boundary conditions for first-order waves are

$$
\begin{gathered}
\frac{\partial^{2} \Phi_{1}^{(1)}}{\partial x^{2}}+\frac{\partial^{2} \Phi_{1}^{(1)}}{\partial z^{2}}=0, \\
\frac{\partial^{2} \Phi_{2}^{(1)}}{\partial x^{2}}+\frac{\partial^{2} \Phi_{2}^{(1)}}{\partial z^{2}}=0, \\
\frac{\partial \Phi_{1}^{(1)}}{\partial z}-\frac{\partial \eta_{I}^{(1)}}{\partial t}=0 \quad \text { at } z=0, \\
\frac{\partial \Phi_{2}^{(1)}}{\partial z}-\frac{\partial \eta_{I}^{(1)}}{\partial t}=0 \quad \text { at } z=0, \\
\rho_{r}\left[g \eta_{I}^{(1)}+\frac{\partial \Phi_{1}^{(1)}}{\partial t}\right]-\left[g \eta_{I}^{(1)}+\frac{\partial \Phi_{2}^{(1)}}{\partial t}\right]=0 \quad \text { at } z=0, \\
\frac{\partial \Phi_{1}^{(1)}}{\partial z}-\frac{\partial \eta_{S}^{(1)}}{\partial t}=0 \quad \text { at } z=h_{1}, \\
g \eta_{S}^{(1)}+\frac{\partial \Phi_{1}^{(1)}}{\partial t}=0 \quad \text { at } z=h_{1}, \\
\frac{\partial \Phi_{2}^{(1)}}{\partial z}=0 \quad \text { at } z=-h_{2} .
\end{gathered}
$$

The first-order solutions are assumed to be

$$
\begin{gathered}
\eta_{I}^{(1)}=\operatorname{Re}\left[\sum_{j=m, n} a_{j} e^{i \theta_{j}}\right], \\
\eta_{S}^{(1)}=\operatorname{Re}\left[\sum_{j=m, n} d_{j} e^{i \theta_{j}}\right], \\
\Phi_{1}^{(1)}=\operatorname{Im}\left\{\sum_{j=m, n}\left[A_{j} \cosh \left(k_{j} z\right)+B_{j} \sinh \left(k_{j} z\right)\right] \cdot e^{i \theta_{j}}\right\},
\end{gathered}
$$

$$
\Phi_{2}^{(1)}=\operatorname{Im}\left\{\sum_{j=m, n} C_{j} \cosh \left[k_{j}\left(z+h_{2}\right)\right] \cdot e^{i \theta_{j}}\right\},
$$

where $\theta_{j}=k_{j} x-\sigma_{j} t+\psi_{j}\left(\psi_{j}\right.$ is the phase $)$ and Re and Im indicate the real and imaginary parts of the complex, respectively. The assumed velocity potentials in (20) and (21) have automatically satisfied boundary conditions (10), (11), and (17). Inserting (18) to (21) into (12) to (16) results in the following relations between the assumed coefficients:

$$
\begin{gathered}
A_{j}=\frac{a_{j}}{\sigma_{j}}\left[\left(1-\frac{1}{\rho_{r}}\right) g+\frac{\sigma_{j}^{2}}{\rho_{r} k_{j} \tanh \left(k_{j} h_{2}\right)}\right], \\
B_{j}=\frac{\sigma_{j} a_{j}}{k_{j}}, \\
C_{j}=\frac{\sigma_{j} a_{j}}{k_{j} \sinh \left(k_{j} h_{2}\right)}, \\
d_{j}=\frac{\sigma_{j}^{2}}{\sigma_{j}^{2} \cosh \left(k_{j} h_{1}\right)-g k_{j} \sinh \left(k_{j} h_{1}\right)} \cdot a_{j}, \\
\sigma_{j}^{4}\left[\operatorname{coth}\left(k_{j} h_{1}\right) \operatorname{coth}\left(k_{j} h_{2}\right)+\rho_{r}\right] \\
-\sigma_{j}^{2} g k_{j}\left[\operatorname{coth}\left(k_{j} h_{1}\right)+\operatorname{coth}\left(k_{j} h_{2}\right)\right] \\
+\left(1-\rho_{r}\right) g^{2} k_{j}^{2}=0 .
\end{gathered}
$$

Equation (26) is the dispersion relation in which two solutions of $\sigma_{j}^{2}$ with a given wavenumber $k_{j}$ exist. It usually refers to the fast-mode and slow-mode solutions. For a case with a very small density difference $\left(\rho_{2}-\rho_{1} \ll \rho_{2}\right)$, the two solutions of $\sigma_{j}^{2}$ are shown below:

$\sigma_{j}^{2}=g k_{j} \tanh \left[k_{j}\left(h_{1}+h_{2}\right)\right], \quad$ for the fast-mode motion,

$$
\sigma_{j}^{2}=g k_{j} \cdot \frac{1-\rho_{r}}{\operatorname{coth}\left(k_{j} h_{1}\right)+\operatorname{coth}\left(k_{j} h_{2}\right)},
$$

for the slow-mode motion.

The slow-mode motion will be excited when the density difference tends to zero. In this mode, the free surface displacement will be very small in comparison with the internal wave displacement. Therefore, the free surface boundary was usually replaced by a rigid lid in many studies for 
simplification. Subsequently, the governing equations and boundary conditions at the second order are shown as

$$
\begin{aligned}
& \frac{\partial^{2} \Phi_{1}^{(2)}}{\partial x^{2}}+\frac{\partial^{2} \Phi_{1}^{(2)}}{\partial z^{2}}=0, \\
& \frac{\partial^{2} \Phi_{2}^{(2)}}{\partial x^{2}}+\frac{\partial^{2} \Phi_{2}^{(2)}}{\partial z^{2}}=0, \\
& \frac{\partial \Phi_{1}^{(2)}}{\partial z}-\frac{\partial \eta_{I}^{(2)}}{\partial t}=-\frac{\partial^{2} \Phi_{1}^{(1)}}{\partial z^{2}} \eta_{I}^{(1)} \\
& +\frac{\partial \Phi_{1}^{(1)}}{\partial x} \frac{\partial \eta_{I}^{(1)}}{\partial x} \quad \text { at } z=0, \\
& \frac{\partial \Phi_{2}^{(2)}}{\partial z}-\frac{\partial \eta_{I}^{(2)}}{\partial t}=-\frac{\partial^{2} \Phi_{2}^{(1)}}{\partial z^{2}} \eta_{I}^{(1)} \\
& +\frac{\partial \Phi_{2}^{(1)}}{\partial x} \frac{\partial \eta_{I}^{(1)}}{\partial x} \quad \text { at } z=0, \\
& \rho_{r}\left[g \eta_{I}^{(2)}+\frac{\partial \Phi_{1}^{(2)}}{\partial t}\right]-\left[g \eta_{I}^{(2)}+\frac{\partial \Phi_{2}^{(2)}}{\partial t}\right] \\
& =-\rho_{r}\left[\frac{\partial^{2} \Phi_{1}^{(1)}}{\partial t \partial z} \eta_{I}^{(1)}+\frac{1}{2}\left(\frac{\partial \Phi_{1}^{(1)}}{\partial x}\right)^{2}+\frac{1}{2}\left(\frac{\partial \Phi_{1}^{(1)}}{\partial z}\right)^{2}\right] \\
& +\left[\frac{\partial^{2} \Phi_{2}^{(1)}}{\partial t \partial z} \eta_{I}^{(1)}+\frac{1}{2}\left(\frac{\partial \Phi_{2}^{(1)}}{\partial x}\right)^{2}\right. \\
& \left.+\frac{1}{2}\left(\frac{\partial \Phi_{2}^{(1)}}{\partial z}\right)^{2}\right] \quad \text { at } z=0 \text {, } \\
& \frac{\partial \Phi_{1}^{(2)}}{\partial z}-\frac{\partial \eta_{S}^{(2)}}{\partial t}=-\frac{\partial^{2} \Phi_{1}^{(1)}}{\partial z^{2}} \eta_{S}^{(1)} \\
& +\frac{\partial \Phi_{1}^{(1)}}{\partial x} \frac{\partial \eta_{S}^{(1)}}{\partial x} \quad \text { at } z=h_{1}, \\
& g \eta_{S}^{(2)}+\frac{\partial \Phi_{1}^{(2)}}{\partial t}=-\frac{\partial^{2} \Phi_{1}^{(1)}}{\partial t \partial z} \eta_{S}^{(1)} \\
& -\frac{1}{2}\left(\frac{\partial \Phi_{1}^{(1)}}{\partial x}\right)^{2}-\frac{1}{2}\left(\frac{\partial \Phi_{1}^{(1)}}{\partial z}\right)^{2} \text { at } z=h_{1}, \\
& \frac{\partial \Phi_{2}^{(2)}}{\partial z}=0 \quad \text { at } z=-h_{2} .
\end{aligned}
$$

The second-order components generated by two first-order $m$ - and $n$-waves are assumed as

$$
\begin{aligned}
& \eta_{I}^{(2)}=\operatorname{Re}\left[\sum_{m, n} a_{m n}^{ \pm} e^{i \theta_{m n}^{ \pm}}\right], \\
& \eta_{S}^{(2)}=\operatorname{Re}\left[\sum_{m, n} d_{m n}^{ \pm} e^{i \theta_{m n}^{ \pm}}\right],
\end{aligned}
$$

$$
\begin{gathered}
\Phi_{1}^{(2)}=\operatorname{Im}\left\{\sum_{m, n}\left[A_{m n}^{ \pm} \cosh \left(k_{m n}^{ \pm} z\right)+B_{m n}^{ \pm} \sinh \left(k_{m n}^{ \pm} z\right)\right]\right. \\
\left.\cdot e^{i \theta_{m n}^{ \pm}}\right\}, \\
\Phi_{2}^{(2)}=\operatorname{Im}\left\{\sum_{m, n}\left[C_{m n}^{ \pm} \cosh \left[k_{m n}^{ \pm}\left(z+h_{2}\right)\right] \cdot e^{i \theta_{m n}^{ \pm}}\right]\right\},
\end{gathered}
$$

where $\theta_{m n}^{ \pm}=k_{m n}^{ \pm} x-\sigma_{m n}^{ \pm} t+\psi_{m n}^{ \pm}, k_{m n}^{ \pm}=k_{m} \pm k_{n}, \sigma_{m n}^{ \pm}=$ $\sigma_{m} \pm \sigma_{n}, \psi_{m n}^{ \pm}=\psi_{m} \pm \psi_{n}$, and the superscripts plus and minus, respectively, denote the superharmonic and subharmonic components, respectively. By inserting (36) into (30) to (34), $a_{m n}^{ \pm}, d_{m n}^{ \pm}, A_{m n}^{ \pm}, B_{m n}^{ \pm}$, and $C_{m n}^{ \pm}$are solved to be

$$
\begin{aligned}
& A_{m n}^{ \pm}=\frac{1}{\Delta^{ \pm}}\left\{\left[\left(\sigma_{m n}^{ \pm}\right)^{2}-g k_{m n}^{ \pm} \mathrm{Ct}_{1}^{ \pm}\right]\right. \\
& \cdot\left[\left(\sigma_{m n}^{ \pm}\right)^{2}\left(k_{m n}^{ \pm}\right)^{-1} \mathrm{Ct}_{2}^{ \pm}\left(\chi_{2}-\chi_{1}\right)\right. \\
& \left.+\left(1-\rho_{r}\right) g \chi_{1}-\sigma_{m n}^{ \pm} \chi_{3}^{ \pm}\right] \\
& +\left(\mathrm{Sh}_{1}^{ \pm}\right)^{-1}\left[\left(1-\rho_{r}\right) g k_{m n}^{ \pm}-\left(\sigma_{m n}^{ \pm}\right)^{2} \mathrm{Ct}_{2}^{ \pm}\right] \\
& \left.\cdot\left[g \chi_{4}+\sigma_{m n}^{ \pm} \chi_{5}^{ \pm}\right]\right\} \text {, } \\
& B_{m n}^{ \pm}=\frac{1}{\Delta^{ \pm}}\left\{\left[\left(\sigma_{m n}^{ \pm}\right)^{2} \mathrm{Ct}_{1}^{ \pm}-g k_{m n}^{ \pm}\right]\right. \\
& \cdot\left[\left(\sigma_{m n}^{ \pm}\right)^{2}\left(k_{m n}^{ \pm}\right)^{-1} \mathrm{Ct}_{2}^{ \pm}\left(\chi_{1}^{ \pm}-\chi_{2}^{ \pm}\right)\right. \\
& \left.-\left(1-\rho_{r}\right) g \chi_{1}^{ \pm}+\sigma_{m n}^{ \pm} \chi_{3}^{ \pm}\right] \\
& \left.-\rho_{r}\left(\sigma_{m n}^{ \pm}\right)^{2}\left(\mathrm{Sh}_{1}^{ \pm}\right)^{-1}\left[g \chi_{4}^{ \pm}+\sigma_{m n}^{ \pm} \chi_{5}^{ \pm}\right]\right\} \text {, } \\
& C_{m n}^{ \pm}=\frac{\left(\mathrm{Sh}_{2}^{ \pm}\right)^{-1}}{\Delta^{ \pm}} \\
& \cdot\left\{\rho_{r}\left(\sigma_{m n}^{ \pm}\right)^{2}\left(\chi_{2}^{ \pm}-\chi_{1}^{ \pm}\right)\right. \\
& \cdot\left[\left(\sigma_{m n}^{ \pm}\right)^{2}\left(k_{m n}^{ \pm}\right)^{-1}-g \mathrm{Ct}_{1}^{ \pm}\right] \\
& +\left[\left(\sigma_{m n}^{ \pm}\right)^{2} \mathrm{Ct}_{1}^{ \pm}-g\left(k_{m n}^{ \pm}\right)\right] \\
& \text {- }\left[\sigma_{m n}^{ \pm} \chi_{3}^{ \pm}-\left(1-\rho_{r}\right) g \chi_{2}^{ \pm}\right] \\
& \left.-\rho_{r}\left(\sigma_{m n}^{ \pm}\right)^{2}\left(\mathrm{Sh}_{1}^{ \pm}\right)^{-1}\left[g \chi_{4}^{ \pm}+\sigma_{m n}^{ \pm} \chi_{5}^{ \pm}\right]\right\}, \\
& d_{m n}^{ \pm}=\frac{1}{\Delta^{ \pm}}\left\{\left(1-\rho_{r}\right) g k_{m n}^{ \pm}\left(\sigma_{m n}^{ \pm} \mathrm{Ct}_{1}^{ \pm} \chi_{4}^{ \pm}+k_{m n}^{ \pm} \chi_{5}^{ \pm}\right)\right. \\
& +\left(\mathrm{Ch}_{1}^{ \pm} \mathrm{Ct}_{1}^{ \pm}-\mathrm{Sh}_{1}^{ \pm}\right) \\
& \cdot\left[\left(\left(\sigma_{m n}^{ \pm}\right)^{2} \mathrm{Ct}_{2}^{ \pm}-\left(1-\rho_{r}\right) g \sigma_{m n}^{ \pm} k_{m n}^{ \pm}\right) \chi_{1}^{ \pm}\right. \\
& \left.-\left(\sigma_{m n}^{ \pm}\right)^{3} \mathrm{Ct}_{2}^{ \pm} \chi_{2}^{ \pm}+\left(\sigma_{m n}^{ \pm}\right)^{2} k_{m n}^{ \pm} \chi_{3}^{ \pm}\right] \\
& -\left(\sigma_{m n}^{ \pm}\right)^{2}\left[\sigma_{m n}^{ \pm}\left(\rho_{r}+\mathrm{Ct}_{1}^{ \pm} \mathrm{Ct}_{2}^{ \pm}\right) \chi_{4}^{ \pm}\right. \\
& \left.\left.+k_{m n}^{ \pm}\left(\rho_{r} \mathrm{Ct}_{1}^{ \pm}+\mathrm{Ct}_{2}^{ \pm}\right) \chi_{5}^{ \pm}\right]\right\} \text {, }
\end{aligned}
$$




$$
\begin{aligned}
a_{m n}^{ \pm}=\frac{1}{\Delta^{ \pm}}\{ & \rho_{r} \sigma_{m n}^{ \pm}\left[g k_{m n}^{ \pm} \mathrm{Ct}_{1}^{ \pm}-\left(\sigma_{m n}^{ \pm}\right)^{2}\right] \chi_{1}^{ \pm} \\
& +\left(\sigma_{m n}^{ \pm} \mathrm{Ct}_{2}^{ \pm} \chi_{2}^{ \pm}-k_{m n}^{ \pm} \chi_{3}^{ \pm}\right) \\
& \cdot\left[g k_{m n}^{ \pm}-\left(\sigma_{m n}^{ \pm}\right)^{2} \mathrm{Ct}_{1}^{ \pm}\right] \\
& \left.-\rho_{r} \sigma_{m n}^{ \pm} k_{m n}^{ \pm}\left(\mathrm{Sh}_{1}^{ \pm}\right)^{-1}\left(g \chi_{4}^{ \pm}+\sigma_{m n}^{ \pm} \chi_{5}^{ \pm}\right)\right\},
\end{aligned}
$$

in which

$$
\begin{aligned}
& \chi_{1}^{ \pm}=-\frac{a_{m} a_{n}}{2} k_{m n}^{ \pm} \\
& \left\{\left[\frac{\left(1-\rho_{r}^{-1}\right) g k_{m}}{\sigma_{m}}+\frac{\sigma_{m}}{\rho_{r}} \operatorname{coth}\left(k_{m} h_{2}\right)\right]\right. \\
& \left.+\left[\frac{\left(1-\rho_{r}^{-1}\right) g k_{n}}{\sigma_{n}}+\frac{\sigma_{n}}{\rho_{r}} \operatorname{coth}\left(k_{n} h_{2}\right)\right]\right\}, \\
& \chi_{2}^{ \pm}=-\frac{a_{m} a_{n}}{2} k_{m n}^{ \pm}\left[\sigma_{m} \operatorname{coth}\left(k_{m} h_{2}\right)+\sigma_{n} \operatorname{coth}\left(k_{n} h_{2}\right)\right] \text {, } \\
& \chi_{3}^{ \pm}=\frac{a_{m} a_{n}}{2} \rho_{r} \\
& \left\{\sigma_{m}^{2}+\sigma_{n}^{2} \pm \sigma_{m} \sigma_{n}\right. \\
& +\left[\frac{\left(1-\rho_{r}^{-1}\right) g k_{m}}{\sigma_{m}}+\frac{\sigma_{m}}{\rho_{r}} \operatorname{coth}\left(k_{m} h_{2}\right)\right] \\
& \left.\cdot\left[\frac{\left(1-\rho_{r}^{-1}\right) g k_{n}}{\sigma_{n}}+\frac{\sigma_{n}}{\rho_{r}} \operatorname{coth}\left(k_{n} h_{2}\right)\right]\right\} \\
& -\frac{a_{m} a_{n}}{2}\left\{\sigma_{m}^{2}+\sigma_{n}^{2}-\frac{\sigma_{m} \sigma_{n}}{\sinh \left(k_{m} h_{2}\right) \sinh \left(k_{n} h_{2}\right)}\right. \\
& \left.\cdot \cosh \left(k_{m n}^{\mp} h_{2}\right)\right\} \text {, } \\
& \chi_{4}^{ \pm}=-\frac{a_{m} a_{n}}{2} k_{m n}^{ \pm} \\
& \cdot\left\{\frac{\sigma_{n}^{2}}{\sigma_{n}^{2} \cosh \left(k_{n} h_{1}\right)-g k_{n} \sinh \left(k_{n} h_{1}\right)}\right. \\
& {\left[\left(\frac{\left(1-\rho_{r}^{-1}\right) g k_{m}}{\sigma_{m}}+\frac{\sigma_{m}}{\rho_{r}} \operatorname{coth}\left(k_{m} h_{2}\right)\right)\right.} \\
& \cdot \cosh \left(k_{m} h_{1}\right)+\sigma_{m} \sinh \left(k_{m} h_{1}\right) \\
& +\frac{\sigma_{m}^{2}}{\sigma_{m}^{2} \cosh \left(k_{m} h_{1}\right)-g k_{m} \sinh \left(k_{m} h_{1}\right)} \\
& \cdot\left[\left(\frac{\left(1-\rho_{r}^{-1}\right) g k_{n}}{\sigma_{n}}+\frac{\sigma_{n}}{\rho_{r}} \operatorname{coth}\left(k_{n} h_{2}\right)\right)\right. \\
& \left.\left.\cdot \cosh \left(k_{n} h_{1}\right)+\sigma_{n} \sinh \left(k_{n} h_{1}\right)\right]\right\} \text {, }
\end{aligned}
$$

$$
\begin{aligned}
& \chi_{5}^{ \pm}=\frac{a_{m} a_{n}}{2} \\
& \cdot\left\{\frac{\sigma_{n}^{2}}{\sigma_{n}^{2} \cosh \left(k_{n} h_{1}\right)-g k_{n} \sinh \left(k_{n} h_{1}\right)}\right. \\
& \cdot\left[\left(\left(1-\rho_{r}^{-1}\right) g k_{m}+\frac{\sigma_{m}^{2}}{\rho_{r}} \operatorname{coth}\left(k_{m} h_{2}\right)\right)\right. \\
& \left.\cdot \sinh \left(k_{m} h_{1}\right)+\sigma_{m}^{2} \cosh \left(k_{m} h_{1}\right)\right] \\
& +\frac{\sigma_{m}^{2}}{\sigma_{m}^{2} \cosh \left(k_{m} h_{1}\right)-g k_{m} \sinh \left(k_{m} h_{1}\right)} \\
& \cdot\left[\left(\left(1-\rho_{r}^{-1}\right) g k_{n}+\frac{\sigma_{n}^{2}}{\rho_{r}} \operatorname{coth}\left(k_{n} h_{2}\right)\right)\right. \\
& \left.\cdot \sinh \left(k_{n} h_{1}\right)+\sigma_{n}^{2} \cosh \left(k_{n} h_{1}\right)\right] \\
& -\left[\left(\frac{\left(1-\rho_{r}^{-1}\right) g k_{m}}{\sigma_{m}}+\frac{\sigma_{m}}{\rho_{r}} \operatorname{coth}\left(k_{m} h_{2}\right)\right)\right. \\
& \cdot\left(\frac{\left(1-\rho_{r}^{-1}\right) g k_{n}}{\sigma_{n}}+\frac{\sigma_{n}}{\rho_{r}} \operatorname{coth}\left(k_{n} h_{2}\right)\right) \\
& \left.\mp \sigma_{m} \sigma_{n}\right] \mathrm{Ch}_{1}^{\mp} \\
& -\left[\left(\frac{\left(1-\rho_{r}^{-1}\right) g k_{n}}{\sigma_{n}}+\frac{\sigma_{n}}{\rho_{r}} \operatorname{coth}\left(k_{n} h_{2}\right)\right) \sigma_{m} \mp \sigma_{n}\right. \\
& \left.\cdot\left(\frac{\left(1-\rho_{r}^{-1}\right) g k_{m}}{\sigma_{m}}+\frac{\sigma_{m}}{\rho_{r}} \operatorname{coth}\left(k_{m} h_{2}\right)\right)\right] \\
& \left.\cdot \mathrm{Sh}_{1}^{\mp}\right\}
\end{aligned}
$$

where

$$
\begin{aligned}
& \Delta^{ \pm}=\left(\sigma_{m n}^{ \pm}\right)^{4}\left(\rho_{r}+\mathrm{Ct}_{1}^{ \pm} \mathrm{Ct}_{2}^{ \pm}\right) \\
&-\left(\sigma_{m n}^{ \pm}\right)^{2} g k_{m n}^{ \pm}\left(\mathrm{Ct}_{1}^{ \pm}+\mathrm{Ct}_{2}^{ \pm}\right)+\left(1-\rho_{r}\right) g^{2}\left(k_{m n}^{ \pm}\right)^{2}, \\
& \mathrm{Sh}_{i}^{ \pm} \equiv \sinh \left(k_{m n}^{ \pm} h_{i}\right), \\
& \mathrm{Ch}_{i}^{ \pm} \equiv \cosh \left(k_{m n}^{ \pm} h_{i}\right), \\
& \mathrm{Ct}_{i}^{ \pm} \equiv \operatorname{coth}\left(k_{m n}^{ \pm} h_{i}\right) .
\end{aligned}
$$

Present two-dimensional solutions can be readily converted to three-dimensional forms by using the vectors of wavenumbers instead of using the scalar forms that are presented. 
It is also noted that the above solutions can be reduced to most existing results including surface waves in a one-fluid system $\left(\rho_{r}=0\right)$ and internal waves in a two-fluid system $[8,9,12,13]$. In the following analyses, the slow-mode motion is considered, and the density ratio $\rho_{r}$ is assumed to be 0.99 for the purpose of approaching the real circumstance in ocean.

\section{Internal Stokes Wave and Its Convergence}

In this section internal Stokes wave and its convergence are examined. First, the first-order solution is assumed as

$$
\eta_{I}^{(1)}=a_{S}^{(1)} \cos (k x-\sigma t)
$$

Based on (41), the second-order solution is solved to be

$$
\begin{aligned}
& \eta_{I}^{(2)}= a_{S}^{(2)} \cos 2(k x-\sigma t) \\
&= a_{S}^{(1)}\left(k a_{S}^{(1)}\right) \cdot \frac{H}{\Delta_{S}} \\
& \cdot\left\{\rho_{r} H\left(\mathrm{Ct}_{1}^{2}-1\right)^{0.5}\right. \\
& \cdot\left[\frac{E \widehat{\mathrm{C}} \mathrm{t}_{1}+1-E H-H \widehat{\mathrm{C}} \mathrm{t}_{1}}{\left(H \widehat{\mathrm{C}} \mathrm{t}_{1}-1\right)}+\frac{\left(E^{2}-1\right)}{2}\right] \\
&+\rho_{r} E\left(2 H-\mathrm{Ct}_{1}\right)+\left(\frac{2 H \mathrm{Ct}}{1}-1\right) \\
&\left.\cdot\left(4 \mathrm{Ct}_{2} \widehat{\mathrm{C}} \mathrm{t}_{2}+3 \rho_{r}+\rho_{r} E^{2}-2+\widehat{\mathrm{C}} \mathrm{t}_{2}^{2}-1\right)\right\} \\
& \equiv \cos 2(k x-\sigma t) \\
& \equiv a_{S}^{(1)}\left(k a_{S}^{(1)}\right) \cdot \mathscr{F}\left(k h_{1}, k h_{2}\right) \cdot \cos 2(k x-\sigma t)
\end{aligned}
$$

where

$$
\begin{aligned}
\mathscr{F}\left(k h_{1}, k h_{2}\right) \equiv & \frac{H}{\Delta_{S}} \\
& \cdot\left\{\rho_{r} H\left(\mathrm{Ct}_{1}^{2}-1\right)^{0.5}\right. \\
& \cdot\left[\frac{E \widehat{\mathrm{C}} \mathrm{t}_{1}+1-E H-H \widehat{\mathrm{C}} \mathrm{t}_{1}}{\left(H \widehat{\mathrm{Ct}}_{1}-1\right)}+\frac{\left(E^{2}-1\right)}{2}\right] \\
& +\rho_{r} E\left(2 H-\mathrm{Ct}_{1}\right)+\left(\frac{2 H \mathrm{Ct}}{4}-1\right) \\
& \left.\cdot\left(4 \mathrm{Ct}_{2} \widehat{\mathrm{C}}_{2}+3 \rho_{r}+\rho_{r} E^{2}-2+\widehat{\mathrm{Ct}}_{2}^{2}-1\right)\right\},
\end{aligned}
$$

$$
\begin{gathered}
\mathrm{Ct}_{i}=\operatorname{coth}\left(2 k h_{i}\right), \\
\widehat{\mathrm{C}} \mathrm{t}_{i}=\operatorname{coth}\left(k h_{i}\right), \\
H=\frac{\sigma^{2}}{g k}, \\
E=\rho_{r}^{-1} \widehat{\mathrm{C}}_{2}+H^{-1}\left(1-\rho_{r}^{-1}\right), \\
\Delta_{S}=4 H^{2}\left(\rho_{r}+\mathrm{Ct}_{1} \mathrm{Ct}_{2}\right) \\
-2 H\left(\mathrm{Ct}_{1}+\mathrm{Ct}_{2}\right)+\left(1-\rho_{r}\right) .
\end{gathered}
$$

The above solution agrees with the theoretical solutions recently given by Umeyama [12] and that obtained earlier by Thorpe [9] (some misprints in (A1.1) of Thorpe's study should be corrected). It is also remarked that the dispersion relation remains unchanged until the third order. Above second-order solution is found to depend only on $k h_{1}, k h_{2}$, and the wave slope $k a_{S}^{(1)}$. Before further investigations are carried out, the convergence for validating the perturbation has to be determined. Though the convergence for surface Stokes waves had been examined by Levi-Civita [15], such an examination for convergence has not yet been well studied for internal Stokes wave. To meet the convergence, the amplitude of second-order wave is required to be less than that of firstorder wave, that is,

$$
\left|k a_{S}^{(1)} \cdot \mathscr{F}\left(k h_{1}, k h_{2}\right)\right|<1 .
$$

In general, this convergence requires that the wave slope is sufficiently small. As for the detailed observation, the convergence zone determined by (47) for the slow-mode motion is shown in Figure 2. It is seen that larger values of wave slope result in a more rigorous condition between $k h_{1}$ and $k h_{2}$. All of the following analyses in this section should be subject to this convergence condition. Ratios of the second-order amplitude relative to the first-order amplitude are displayed in Figures 3 and 4 . Figure 3 shows the results under different wave slopes. Solid and dash curves represent the results of internal-wave amplitude ratios $a_{S}^{(2)} / a_{S}^{(1)}$ and surface-wave amplitude ratios $d_{S}^{(2)} / d_{S}^{(1)}$, respectively. Waves with larger wave slopes are found to have stronger second-order effects on amplitudes. The effects of the thickness ratio of two fluid layers are shown in Figure 4 . It is found that as values of $h_{1} / h_{2}$ become small enough, the amplitude ratios become negative. Positive values of amplitude ratios imply that the wave crests (with respect to the below fluid) will sharpen and the wave troughs will flatten. Such a phenomenon is analogous to that appearing in a single-fluid system. On the contrary, negative values suggest the occurrence of a sharper trough and a flatter crest (also with respect to the below fluid). Other properties of Stokes waves will be discussed in the next section. 


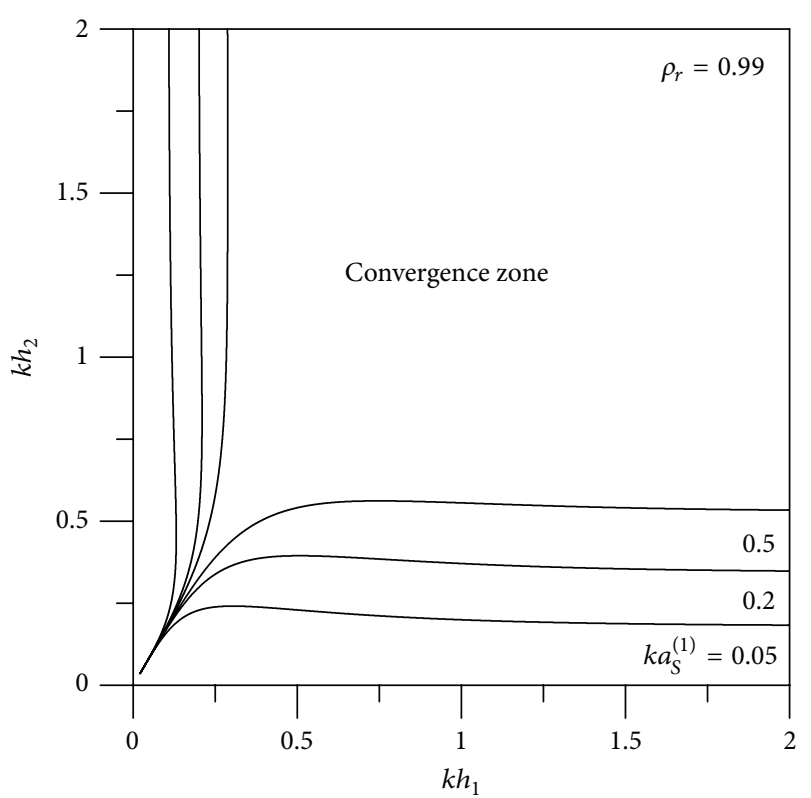

FIGURE 2: Convergence zone for internal Stokes waves with various wave slopes.

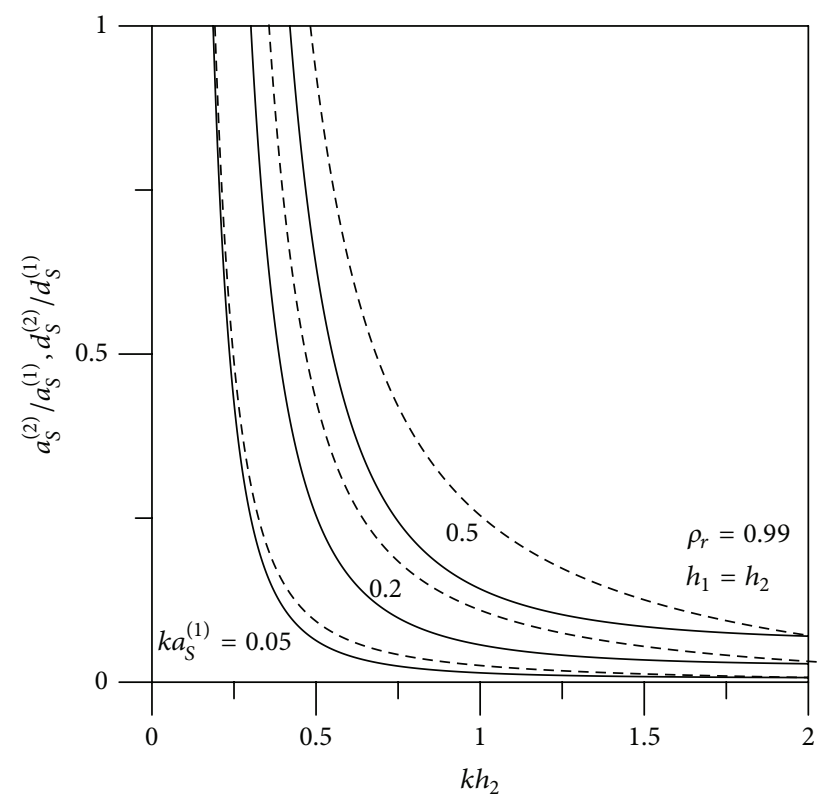

Figure 3: Ratios of the second-order amplitude to the first-order amplitude for Stokes waves with various wave slopes. Solid lines for internal waves and dash lines for surface waves.

\section{Superharmonic and Subharmonic Waves and the Convergence}

Superharmonic and subharmonic interactions of two arbitrary first-order waves ( $m$-wave and $n$-wave) are examined in this section. Because six length parameters, $h_{1}, h_{2}, k_{m}^{-1}$, $k_{n}^{-1}, a_{m}$, and $a_{n}$, appear in the second-order solutions, the condition $a_{m}=a_{n}$, which will cause the strongest interaction,

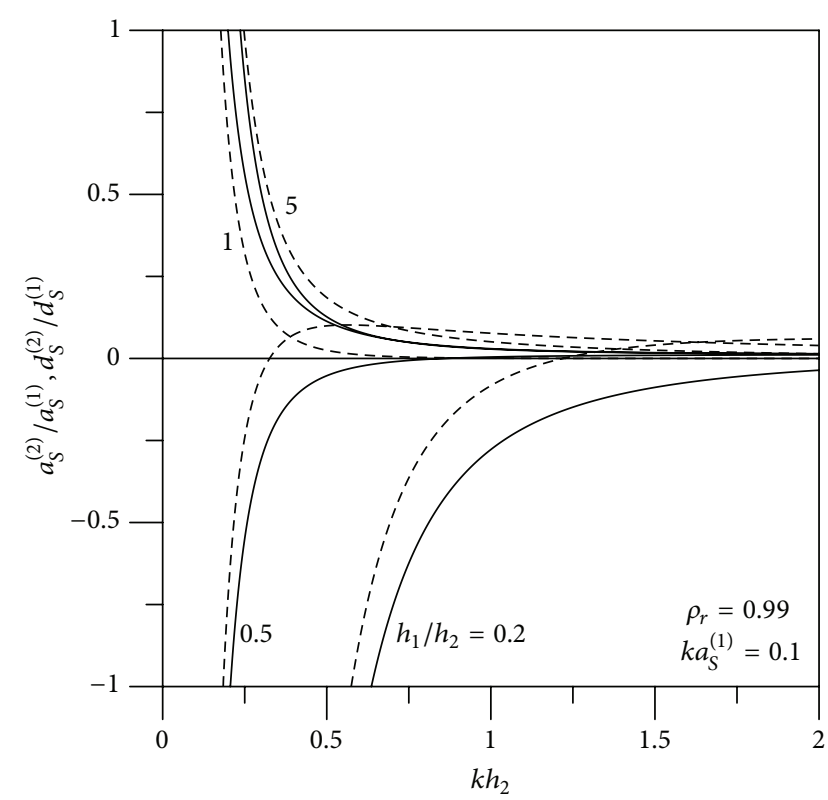

FIgURE 4: Ratios of the second-order amplitude to the first-order amplitude for Stokes waves with various thickness ratios. Solid lines for internal waves and dash lines for surface waves.

is assumed to investigate the effects of two important parameters, $k_{n} / k_{m}$ and $h_{1} / h_{2}$, on second-order wave properties. The convergence is firstly examined. It may require the ratio of the second-order amplitude relative to the summation of two first-order amplitudes to be less than unity:

$$
\left|\frac{a_{m n}^{ \pm}}{a_{m}+a_{n}}\right|<1
$$

For Stokes waves, the convergences defined by (47) and (48) are identical. As for arbitrary wave interactions, Figure 5 shows the convergences under various wavenumber ratios $k_{n} / k_{m}$ for superharmonic (solid lines) and subharmonic (dash line) interactions. It is found that the smaller wavenumber ratio $k_{n} / k_{m}$ results in a more rigorous convergence condition between $k_{m} h_{1}$ and $k_{m} h_{2}$. It implies that when one wave ( $m$-wave) interacts with another shorter wave $(n$ wave), it yields a wider convergence zone. In addition, the convergence for the subharmonic case is more severe than that for the superharmonic case. As mentioned in Section 3, the larger wave slope will narrow the convergence range.

After examining the convergence condition, transfer functions defined to measure the second-order effects on wave profiles are subsequently investigated. The superharmonic and subharmonic transfer functions for surface (with the subscript $S$ ) and internal (with the subscript $I$ ) waves are, respectively, defined as

$$
G_{m n, S}^{ \pm} \equiv \frac{d_{m n}^{ \pm}}{d_{m} d_{n}}, \quad G_{m n, I}^{ \pm} \equiv \frac{a_{m n}^{ \pm}}{a_{m} a_{n}}
$$

Note that the transfer functions are independent of the amplitudes of both first-order waves. Figures 6, 7, and 8 show the behaviors of the superharmonic transfer functions for 


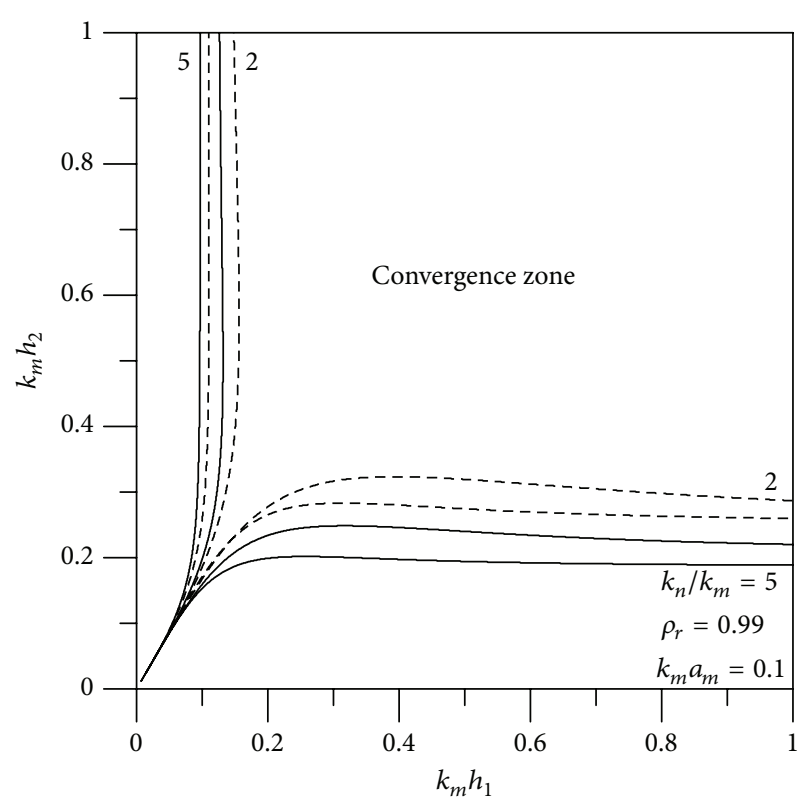

Figure 5: Convergence zone for the super- and the subharmonic interactions with various wavenumber ratios. Solid line for superharmonic interactions and dash lines for subharmonic interactions.

internal and surface waves over the depth range $0<k_{m} h_{2}<5$. All of these figures are plotted under the same condition $h_{1}=h_{2}$. The transfer functions for internal waves with various wavenumber ratios are plotted in Figure 6. A larger value of the wavenumber ratio results in stronger secondorder effects on the wave profile. In addition, the tendency of $G_{m n, I}^{+}$is quite similar to that for the single-fluid case (see Sand and Mansard [6, Figure 2]). Results for surface waves are plotted in Figure 7. The magnitudes of the transfer functions for surface waves are much greater than those for internal waves. It is also found that all transfer functions with different wavenumber ratios are always negative over the whole range of $k_{m} h_{2}$. Figure 8 displays the amplitude ratio of surface waves to internal waves at the first order (dash line) and the second order (solid line). In virtue of only a very small undulation at the free surface for the slow-mode motion, the values of these ratios are multiplied by one thousand for a clearer understanding. It reveals that internal and surface waves are $180^{\circ}$ out of phase for the slow-mode motion. Moreover, a larger amplitude ratio of wavenumbers of the first-order waves causes a faster decay of second-order effects while $k_{m} h_{2}$ grows.

Results for the subharmonic cases are plotted in Figures $9,10,11,12$, and 13. For the condition $h_{1}=h_{2}$, Figure 9 shows that values of transfer functions for internal waves remain negative over the whole range of $k_{m} h_{2}$. The behaviors are analogous to those of the single-fluid case (see Sand [16, Figure 2]). Figure 10 indicates that the transfer functions for surface waves are, respectively, positive and negative in the long-wave and the short-wave regimes. Similar to results for the superharmonic case, the thickness ratios of two fluid layers strongly dominate the signs and magnitudes of the transfer functions, as shown in Figures 11 and 12. Motions of

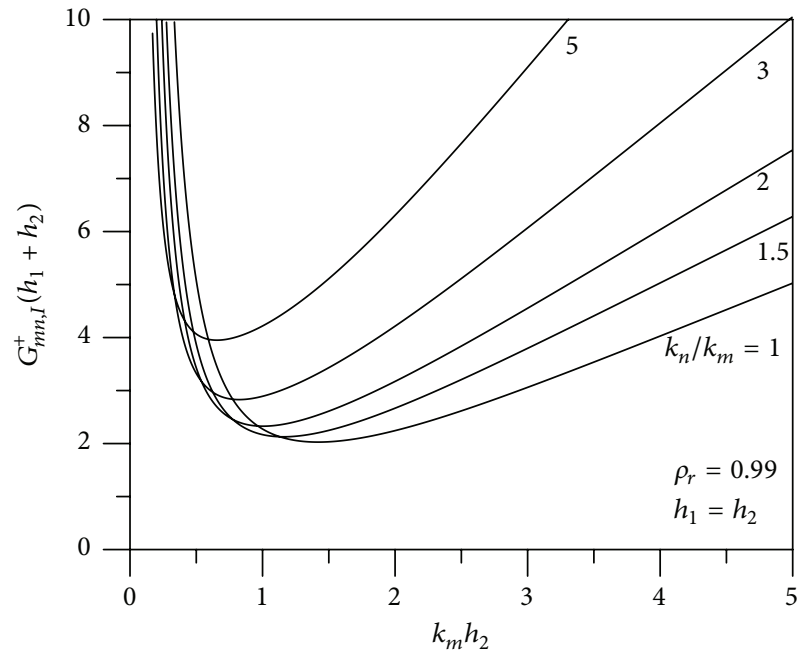

FIGURE 6: Superharmonic transfer functions for internal waves with various wavenumber ratios.

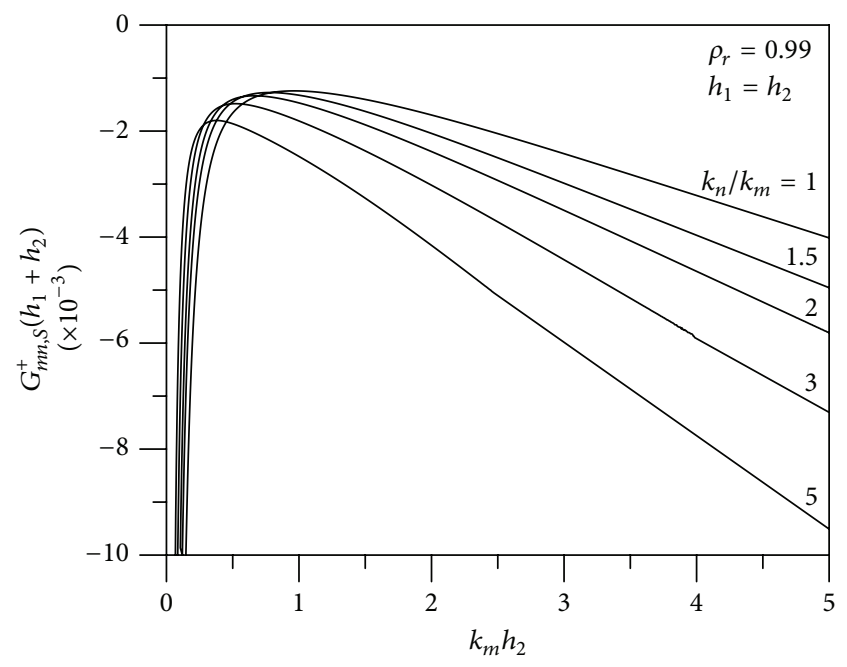

FIGURE 7: Superharmonic transfer functions for surface waves with various wavenumber ratios.

the second-order internal and surface waves could be either in phase or $180^{\circ}$ out of phase depending on the values of $k_{m} h_{2}$, as shown in Figure 13.

Another important wave property, the horizontal and vertical velocities, is also investigated. They can be directly obtained by differentiating the velocity potentials with respect to $x$ and $z$ :

$$
\begin{aligned}
& u_{i}=u_{i}^{(1)}+u_{i}^{(2)}=\frac{\partial}{\partial x}\left(\Phi_{i}^{(1)}+\Phi_{i}^{(2)}\right), \\
& v_{i}=v_{i}^{(1)}+v_{i}^{(2)}=\frac{\partial}{\partial z}\left(\Phi_{i}^{(1)}+\Phi_{i}^{(2)}\right) .
\end{aligned}
$$

Since the phase velocities of all first-order waves and the corresponding second-order wave are different, the phases of $m$-wave, $n$-wave, and the associated second-order wave in (50) are set to be zero $\left(\theta_{m}=\theta_{n}=\theta_{m n}^{ \pm}=0\right)$ 


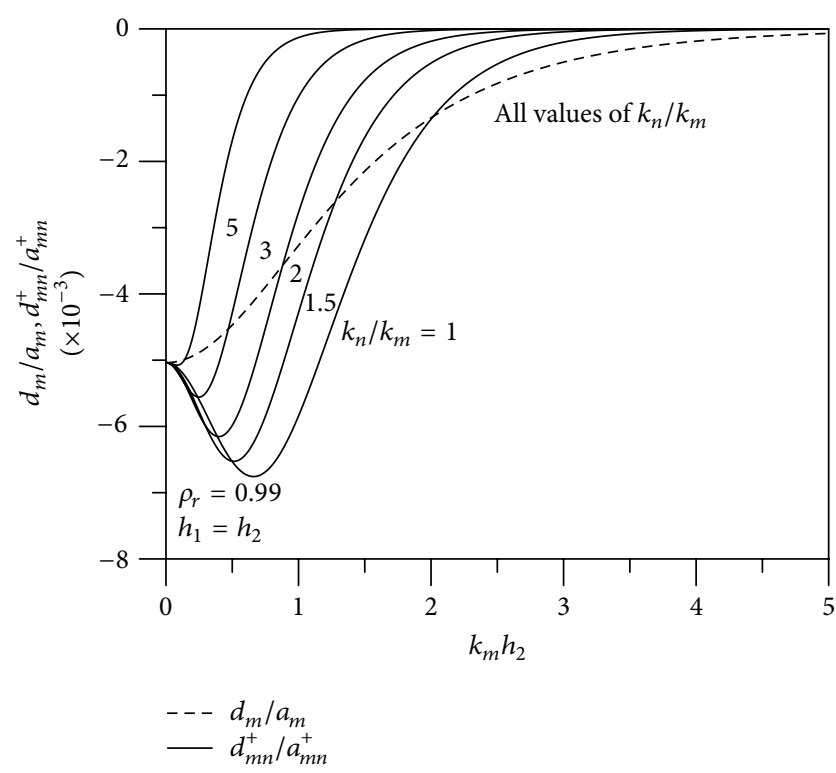

FIGURE 8: Ratios of the amplitude of surface wave to that of internal wave for superharmonic interactions (solid lines for second-order waves with various wavenumbers and dash lines for first-order waves).

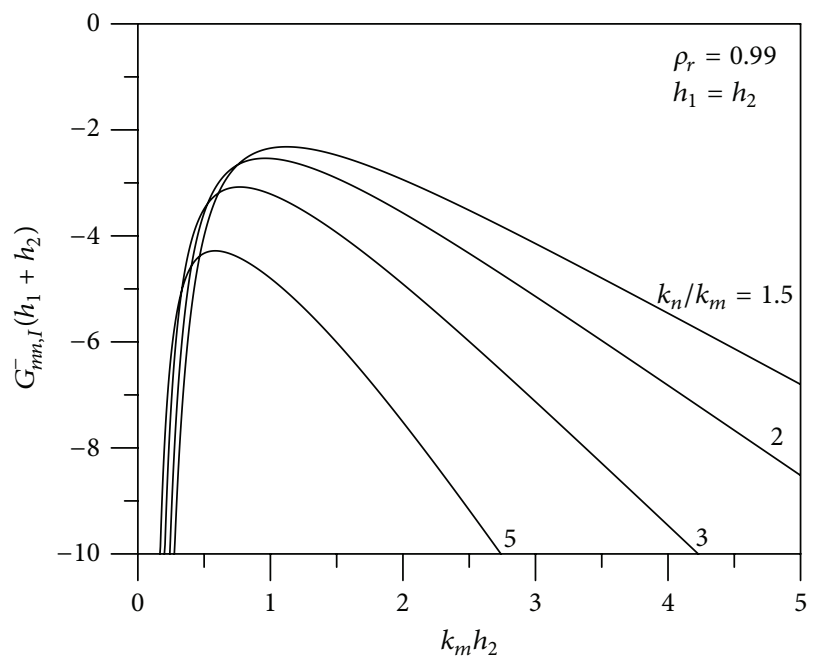

FIGURE 9: Subharmonic transfer functions for internal waves with various wavenumber ratios.

for examination. For the superharmonic case, ratios of the second-order velocity to the first-order velocity with different wavenumber ratios for the shallower-water $\left(k_{m} h_{2}=0.5\right)$ and the deeper-water $\left(k_{m} h_{2}=1.0\right)$ regimes are, respectively, depicted in Figures 14 and 15. Solid lines and dash lines, respectively, stand for the horizontal and vertical velocity ratios along the normalized $z$-axis. In the shallower-water regime, it is also found that the second-order effects are stronger in the upper layer. As for the deeper-water regime, larger wavenumber ratios lead to greater second-order effects near the interface. All second-order effects near the free surface and the bottom are comparatively slight.

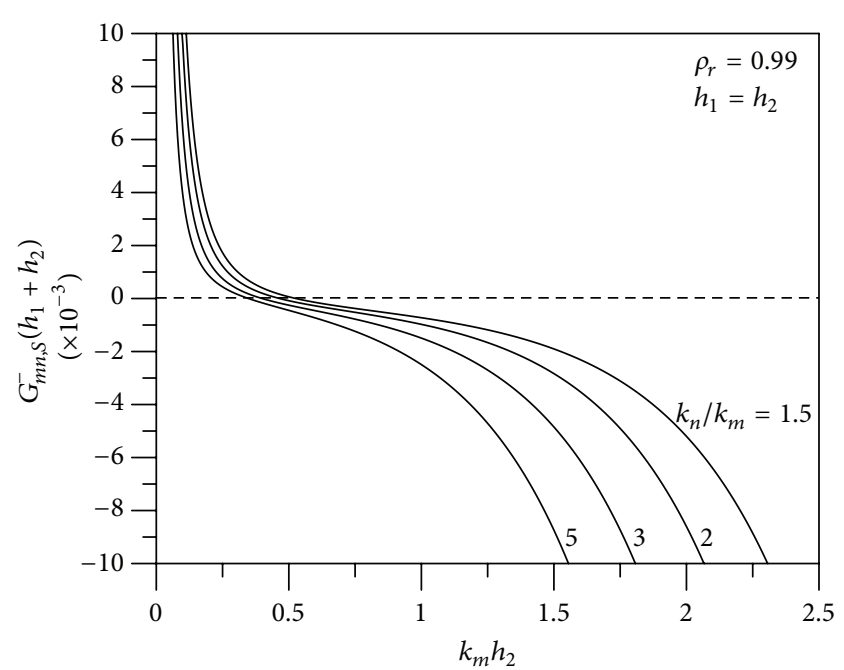

FIGURE 10: Subharmonic transfer functions for surface waves with various wavenumber ratios.

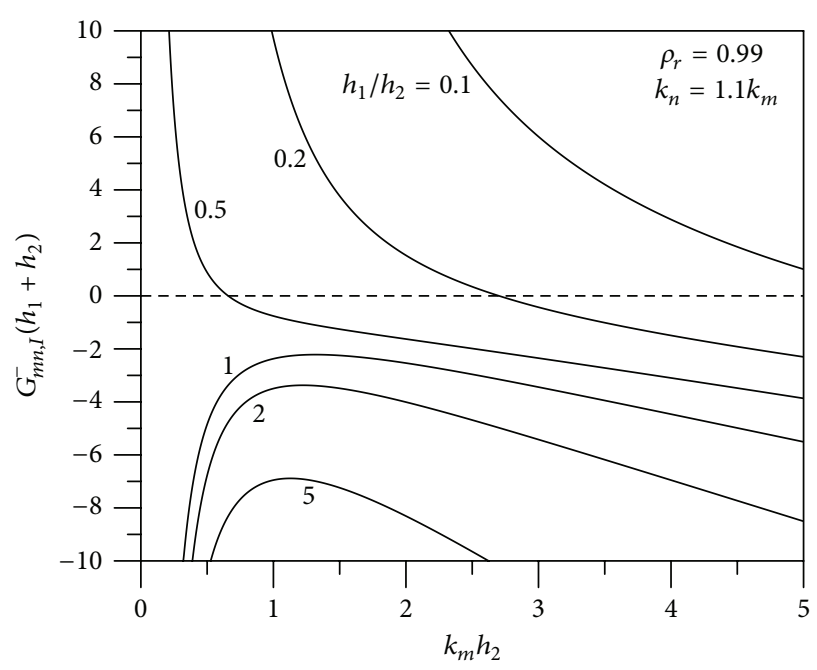

FIGURE 11: Subharmonic transfer functions for internal waves with various thickness ratios.

Results for the subharmonic case are plotted in Figures 16 and 17 for the conditions $k_{m} h_{2}=0.5$ and $k_{m} h_{2}=1.0$, respectively. The results show that second-order effects are stronger in the lower fluid. It is also observed that stronger secondorder effects occur near the interface in the case of larger wavenumber ratios. Moreover, the effects of the thickness ratio $h_{1} / h_{2}$ are not as important as those of the wavenumber ratio $k_{n} / k_{m}$. The reason is that the particle velocities far from the interface are usually smaller in comparison with those near the interface. Hence, the corresponding diagrams for various values of $h_{1} / h_{2}$ are ignored herein.

Finally, we note that particle horizontal velocities of two fluids are identical at the interface due to the boundary conditions, (3) and (4). As viscous effects are not taken into account, particle horizontal velocities of two fluids do not satisfy the continuity requirement at the interface. 


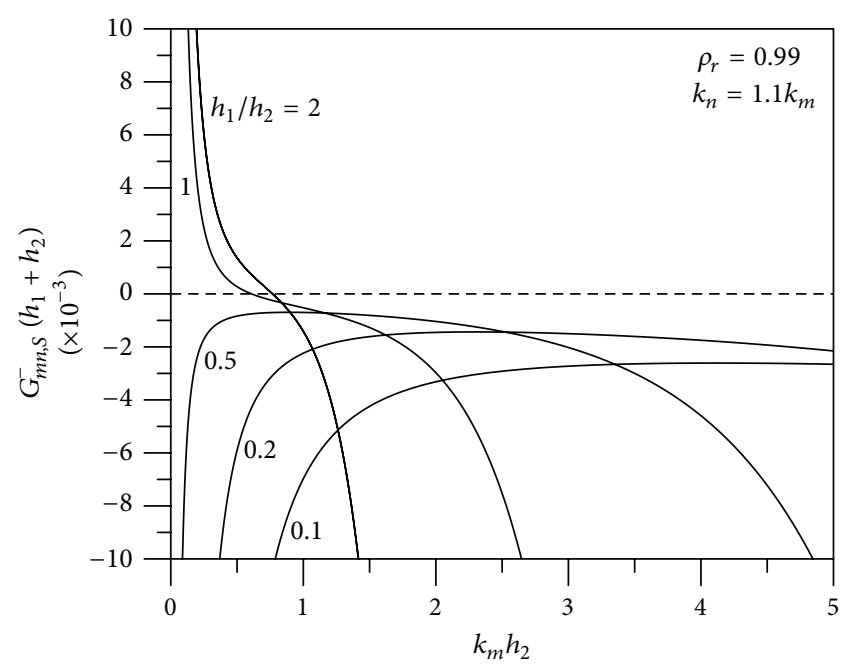

FIGURE 12: Subharmonic transfer functions for surface waves with various thickness ratios.

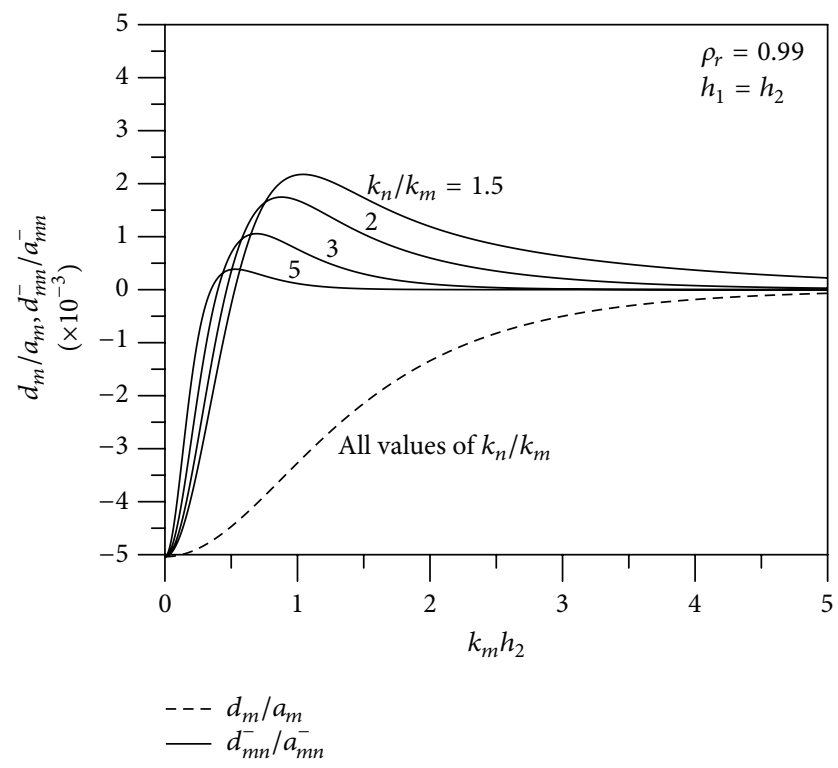

FIGURE 13: Ratios of the amplitude of surface wave to that of internal wave for subharmonic interactions (solid lines for second-order waves with various wavenumbers and dash lines for first-order waves).

\section{Conclusions}

Second-order theory for internal waves in a two-fluid system and the corresponding convergence are presented in this paper. Internal Stokes waves, the second-order interactions of two arbitrary linear waves, the associated convergence condition, and the particle velocities are examined. The crucial conclusions are summarized in the following.

(1) For internal Stokes waves, our second-order solutions are in agreement with those of past studies, and the convergence of the second-order theory is found to depend only on $k h_{1}, k h_{2}$, and the wave slope $k a_{S}^{(1)}$.

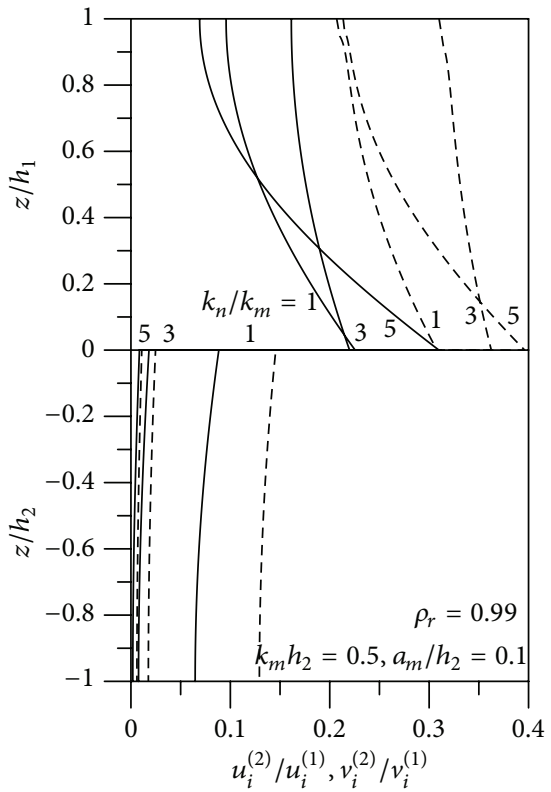

FIGURE 14: Velocity ratios of the second-order waves to the firstorder waves along the $z$-axis for the superharmonic interactions with $k_{m} h_{2}=0.5$. Solid lines for horizontal velocity ratios and dash lines for vertical velocity ratios.

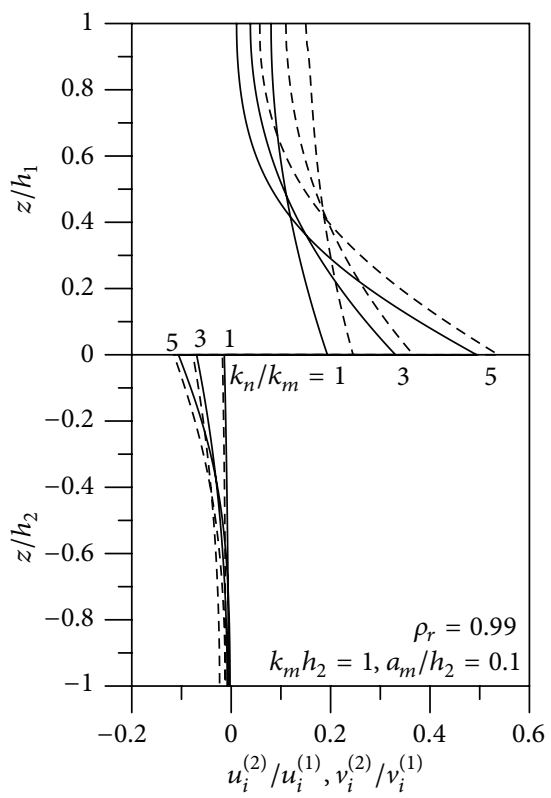

FIGURE 15: Velocity ratios of the second-order waves to the firstorder waves along the $z$-axis for the superharmonic interactions with $k_{m} h_{2}=1.0$. Solid lines for horizontal velocity ratios and dash lines for vertical velocity ratios.

A larger value of wave slope will require a more rigorous convergence condition between $k h_{1}$ and $k h_{2}$. Larger wave slopes also result in stronger secondorder effects either on internal or surface waves. The depth ratio $h_{1} / h_{2}$ is an important parameter for measuring wave profiles influenced by second-order 


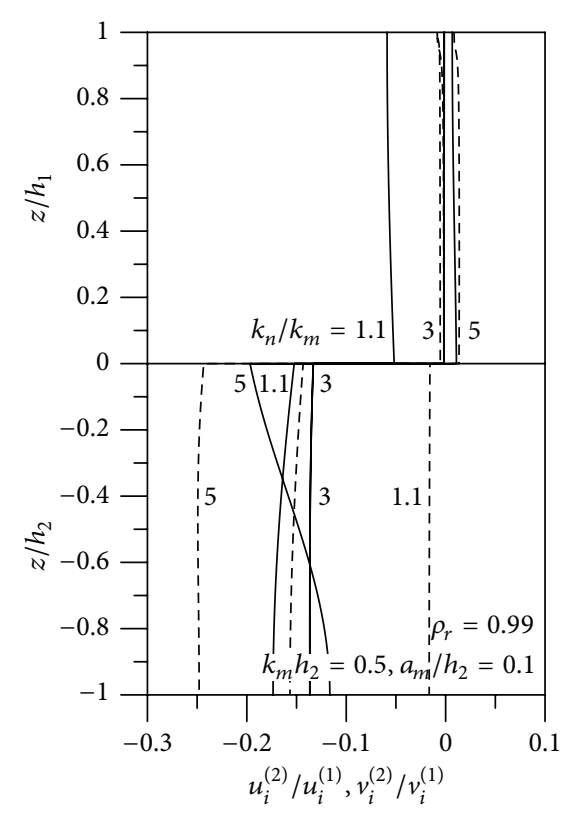

FIGURE 16: Velocity ratios of the second-order waves to the firstorder waves along the $z$-axis for the subharmonic interactions with $k_{m} h_{2}=0.5$. Solid lines for horizontal velocity ratios and dash lines for vertical velocity ratios.

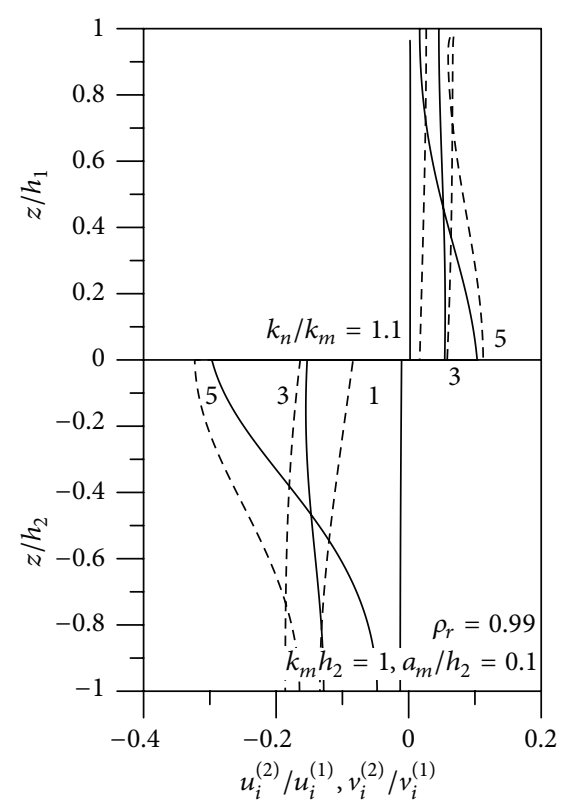

FIgURE 17: Velocity ratios of the second-order waves to the firstorder waves along the $z$-axis for the subharmonic interactions with $k_{m} h_{2}=1.0$. Solid lines for horizontal velocity ratios and dash lines for vertical velocity ratios.

effects. As $h_{1} / h_{2}$ becomes small enough, the internal wave profile will have a sharp trough and a flattened crest with respect to the below fluid. On the contrary, internal waves with sharp crests and flattened troughs will occur as $h_{1} / h_{2}$ becomes large enough.
(2) For second-order interactions arising from two arbitrary waves, a smaller wavenumber ratio $k_{n} / k_{m}$ results in a more rigorous convergence condition between $k_{m} h_{1}$ and $k_{m} h_{2}$. The convergence for the subharmonic interaction is more severe than that for the superharmonic case. In addition, larger values of wave slopes will narrow the convergence range.

(3) Transfer functions measure the magnitude of secondorder effects on wave profiles. For internal waves, transfer functions of all wavenumber ratios respectively remain positive and negative for the superand subharmonic cases. The thickness ratio of the two fluid layers is found to influence not only the magnitude but also the sign of transfer functions. In the long-wave regime, transfer functions usually have opposite signs for the thicker-upper-layer case and the thicker-lower-layer case. Moreover, the firstorder surface and internal waves are $180^{\circ}$ out of phase for the slow-mode motion. The second-order surface and internal waves are also $180^{\circ}$ out of phase for the superharmonic case and are in phase over most of the range of $k_{m} h_{2}$ for the subharmonic case.

(4) As for particle velocities, larger wave number ratios cause stronger second-order effects near the interface. All second-order effects near the free surface and the bottom are much slighter than those near the interface.

Finally, it is noted again that most existing theories for two-fluid and one-fluid systems are included in the present solutions.

\section{Acknowledgments}

The authors appreciate the suggestions and comments from anonymous reviewers. Support from the International Wave Dynamics Research Center of National Cheng Kung University is appreciated. In addition, the research funding provided by the National Science Council of Taiwan with Contracts NSC 101-2221-E-270-001-MY2 and NSC 102-2911-I-006-302 is also acknowledged.

\section{References}

[1] G. G. Stokes, "On the theory of oscillatory waves," Transactions of the Cambridge Philosophical Society, vol. 8, pp. 441-455, 1847.

[2] M. S. Longuet-Higgins and R. W. Stewart, "Radiation stress and mass transport in gravity waves, with application to "surf beats"', Journal of Fluid Mechanics, vol. 13, pp. 481-504, 1962.

[3] M. S. Longuet-Higgins and R. W. Stewart, "Radiation stresses in waterwaves: a physical discussion with applications," Deep Sea Research, vol. 11, pp. 529-562, 1964.

[4] N. E. Ottesen-Hansen, "Long period waves in natural wave trains," Progress Reports 46, Institute of Hydrodynamics and Hydraulic Engineering, Technical University Denmark, Lyngby, Denmark, 1978.

[5] R. G. Dean and N. J. Sharma, "Simulation of wave systems due to nonlinear directional spectra," in Proceedings of International 
Symposium Hydrodynamics in Ocean Engineering, vol. 2, p. 1211, The Norwegian Institute of Technology, 1981.

[6] S. E. Sand and E. P. D. Mansard, "Reproduction of higher harmonics in irregular waves," Ocean Engineering, vol. 13, no. 1, pp. 57-83, 1986.

[7] H. A. Schäffer, "Second-order wave maker theory for irregular waves," Ocean Engineering, vol. 23, pp. 47-88, 1996.

[8] J. N. Hunt, "Interfacial waves of finite amplitude," La Houille Blanche, vol. 16, pp. 515-531, 1961.

[9] S. A. Thorpe, "On the shape of progressive internal waves," Philosophical Transactions of the Royal Society. London A, vol. 263, no. 1145, pp. 563-614, 1968.

[10] Y. Tsuji and Y. Nagata, "Stokes' expansion of internal deep water waves to the fifth order," Journal of the Oceanographical Society of Japan, vol. 29, no. 2, pp. 61-69, 1973.

[11] J. Y. Holyer, "Large amplitude progressive interfacial waves," Journal of Fluid Mechanics, vol. 93, no. 3, pp. 433-448, 1979.

[12] M. Umeyama, "Experimental and theoretical analyses of internal waves of finite amplitude," Journal of Waterway, Port, Coastal and Ocean Engineering, vol. 128, no. 3, pp. 133-141, 2002.

[13] J. B. Song, "Second-order random wave solutions for internal waves in a two-layer fluid," Geophysical Research Letters, vol. 31, no. 15, pp. L15302-L15304, 2004.

[14] C. M. Liu, "Second-order random internal and surface waves in a two-fluid system," Geophysical Research Letters, vol. 33, no. 6, p. L06610, 2006.

[15] T. Levi-Civita, "Détermination rigoureuse des ondes permanentes d'ampleur finie," Mathematische Annalen, vol. 93, no. 1, pp. 264-314, 1925.

[16] S. E. Sand, "Wave grouping described by bounded long waves," Ocean Engineering, vol. 9, no. 6, pp. 567-580, 1982. 


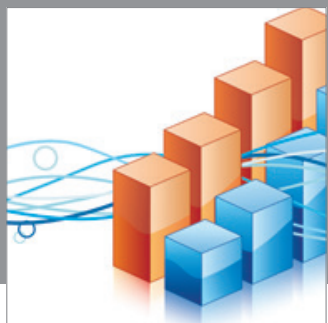

Advances in

Operations Research

mansans

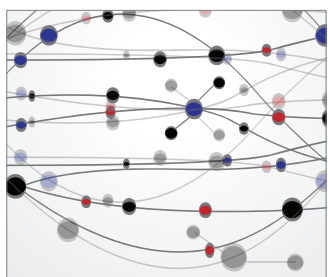

The Scientific World Journal
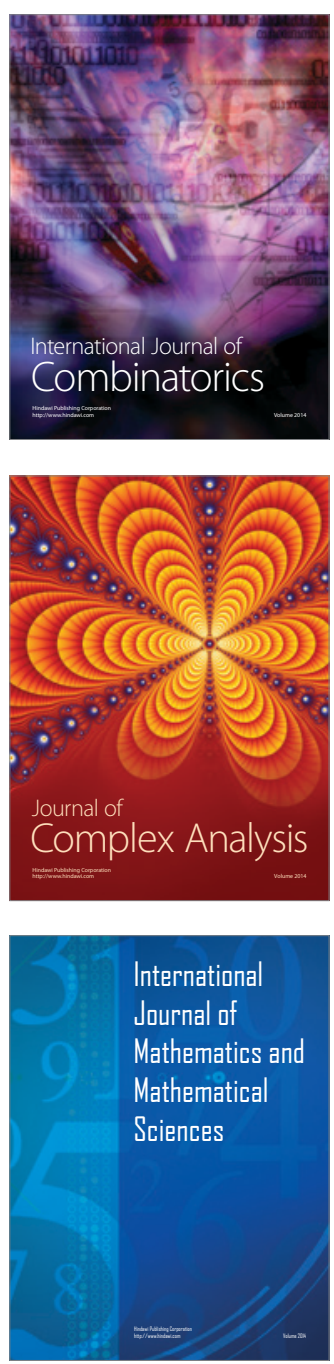
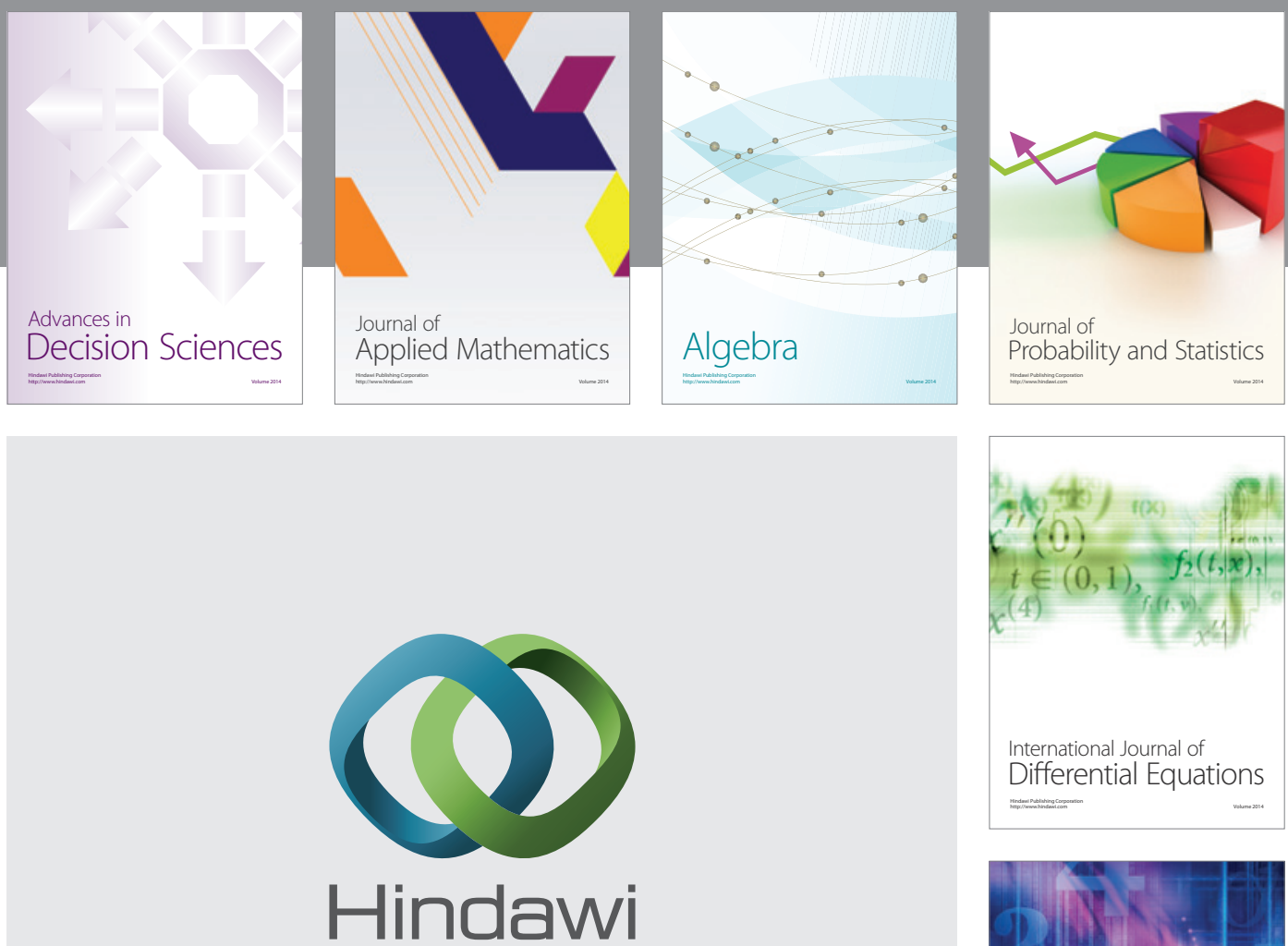

Submit your manuscripts at http://www.hindawi.com
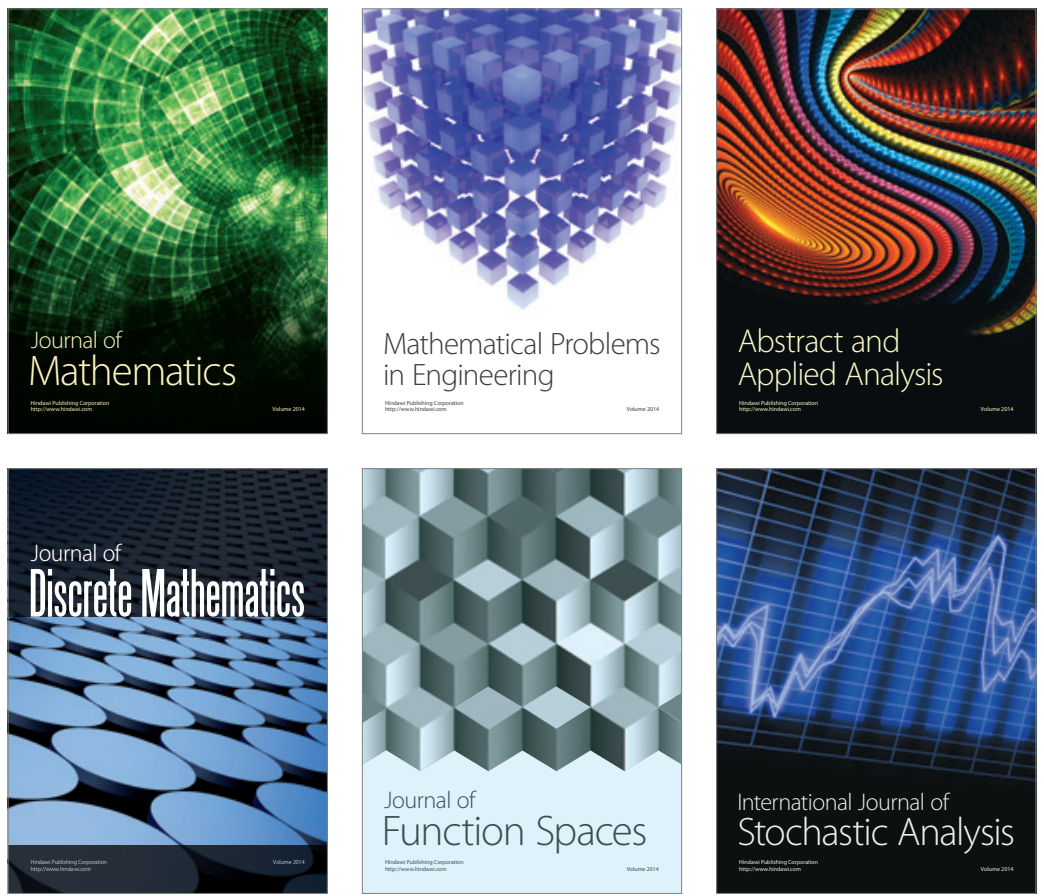

Journal of

Function Spaces

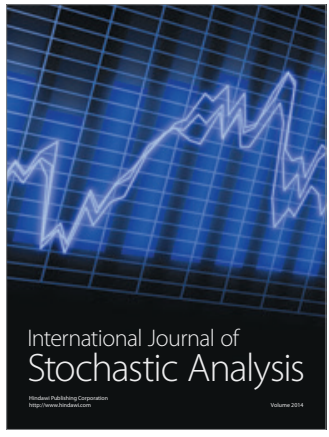

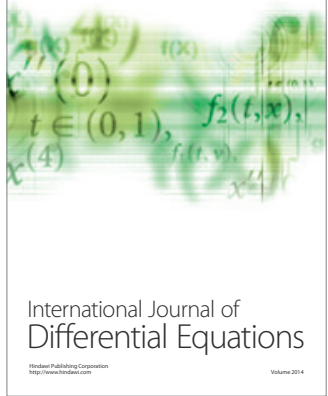
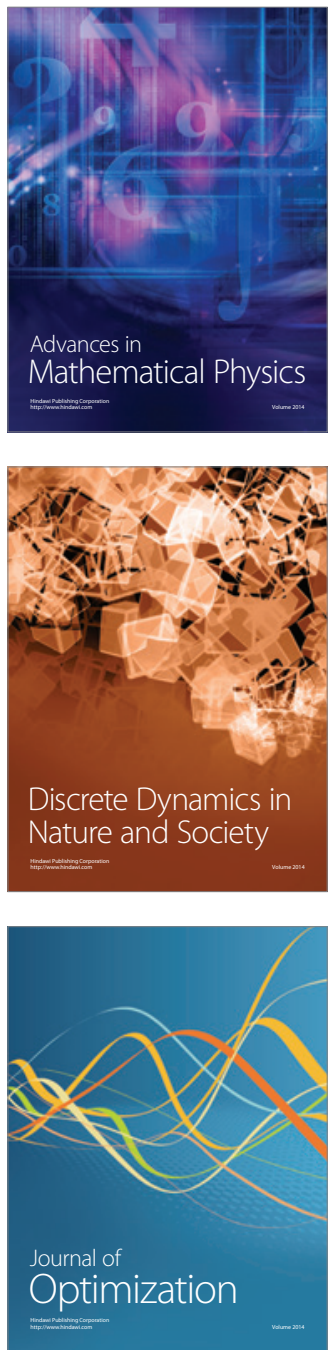